\title{
Nonlinear speed estimation of a GPS-free UAV
}

\author{
G.L Santosuosso* Khadidja Benzemrane ${ }^{\dagger}$ and Gilney Damm ${ }^{\ddagger}$
}

September 26, 2011

\begin{abstract}
In this paper the problem of robust state observer design for a class of Unmanned Aerial Vehicles (UAV) is addressed. A prototype 4 rotors helicopter robot for indoors and outdoors applications is considered: the drone is not equipped with GPS related devices, so that we describe a strategy to estimate its translational velocity vector based on acceleration, angles and angular speeds measurements only. Since the linearized system is non observable at the equilibrium point, a nonlinear observability verification is performed for persistently exciting trajectories. A global exponential solution to this open problem is provided in the framework of adaptive observation theory when exact measurements are available. A modified observer is presented to enhance velocity estimation robustness in the realistic case of noisy measurements. The results are compared with a classical estimation strategy based on the Extended Kalman Filter to test the algorithm's performance.
\end{abstract}

\section{Introduction}

The speed estimation problem has an important role in the context of vehicle control. For land moving robots, the odometry time derivative has a satisfactory performance while for large aerial vehicles (manned or unmanned) velocity estimations can be obtained via approximate derivation of the successive measurements from GPS sensors, motivated by the small resulting errors compared to the measured variables. For fast aircrafts the standard procedure is integrating the acceleration and coupling this result to the derivative of GPS measurements. In fact, signals (translational acceleration, angles and angular velocity) delivered by the embedded Inertial Measurement Unit (IMU) can be used to obtain position information through a double integration process. However, because of sensor drift, such procedure requires high precision IMU. This sensor can't be used for small size UAVs where cost, weight and volume are the most important constraints.

For such applications, two critical issues arise in an "open loop" strategy like direct acceleration integration: an unknown constant estimation error is produced even when exact acceleration measurements are available while a random drift is induced by noisy acceleration estimations. In practice, numerical integration along with measurement noise induces a very fast growing velocity measurement error.

Reasonable approaches to overcome these problems are represented by sensor fusion techniques, in order to compensate the lack of precision of low cost IMUs. In that context the information obtained from the GPS is used to bound the integration error and initial conditions for acceleration integration are provided by GPS devices [29]. This technique provides bounded errors that are related to the GPS order of magnitude precision and sample rate. Currently used GPS may assure a precision of 5-10 meters with a sample rate of 1-2 Hz. The resulting errors are usually small compared to the size of aerial vehicles and the distance to obstacles. In the same way, the control systems usually applied to these vehicles are robust enough to accept the residual disturbance on the speed estimation provided by these methods. Unfortunately, the estimation approach above cannot be implemented on small drones less than 1 meter wide and flying at low speed that we consider in this note. This issue is even more relevant in indoors or simply urban applications as inspecting bridges and industrial facilities which cannot rely on GPS, since satellite's signals are shaded by the inspected structure.

\footnotetext{
*G. Santosuosso is with Dipartimento di Ingegneria Elettronica, Universitá di Roma Tor Vergata, Via del Politecnico 1, 00133 Rome, Italy santosuosso@ing. uniroma2.it

${ }^{\dagger}$ K.Benzemrane is with Laboratoire IBISC - CNRS/Université d'Evry Val d'Essonne, 40 rue du Pelvoux 91020 Evry Cedex, France khadidja.benzemrane@ibisc.fr

${ }^{\ddagger}$ G. Damm is with Laboratoire IBISC - CNRS/Université d'Evry Val d'Essonne, 40 rue du Pelvoux 91020 Evry Cedex, France gilney.damm@ibisc.fr
} 
A technical solution when no obstacles are present would involve the use of a D-GPS system. These systems are known as centimetric GPS and have a precision of some centimeters, but they are very expensive also, heavyweight and low-range operating equipment. In the same way, Doppler measurements coupled with GPS can actually increase velocity estimation accuracy, but they can only be used in special conditions of smoothness and continuity of obstacles, as well as a good knowledge of their position and shape.

In [21], a control law based on computer vision is proposed for quasi-stationary flights above a planar structure like bridges to perform inspection tasks. Likewise, estimates of the pose and velocity of a rigid body can be provided by an algorithm based on IMU data and images provided by a camera (see [5]). The main drawback of this method is the large amount of computer resources needed. It is still not possible to apply vision algorithms in the small micro-controllers used in the considered UAV's. Displacing the image processing algorithms to computers at a land base is hardly implemented for two reasons: first, the time delay induced by remote computing and communication, along with the danger of losing the contact with the drone could inevitably imply disruptive device instability; a second important drawback is the sensibility of cameras and vision algorithms to light variations, associated to outdoor drone displacement. Vision can be used in an hierarchical display where a slow controller gives path planning references to a fast stabilizing one, as in [17], where an Unscented Kalman Filter is applied to the navigation problem and a solution for simultaneous localization and mapping when no GPS signal is available is proposed. The result described in [17] relies on an Inertial Measurement Unit along with a monocular camera only and possible losses of vision or radio contact do not affect the UAV's stability. In the field of fusion techniques, an interesting solution to the problem of estimation of the position and velocity of UAVs is proposed in [27] and [28]. The algorithm based on Kalman filtering techniques uses the magnetic field disturbances to improve the estimation error due to the use of low cost IMU; it may be useful for indoor applications.

In the present paper we consider the problem of translational velocity estimation from measurements of translational accelerations in the local reference frame, Euler angles in the global reference frame and angular speeds in the local reference frame. Since translational velocity knowledge is necessary for an efficient drone control design, most literature about small flying robots control assumes that at least the translational speeds are available (see [9], [10], [15], [24]). This is motivated by the fact that it is hardly possible for a human pilot (inboard or in tele-operation) to regulate a drone by giving references and trajectories as inputs based on the sole acceleration measurement. Notice that in the general case, the reliable estimation of the speed vector is still an open problem. In fact, the acceleration in the local (body fixed) frame is not a standard measurement from IMU, but even if this information is available the classical open loop integration using this information would roughly lead to the same results obtained with the standard acceleration measurement in the global reference frame.

Recently, [4], [29], [30] have proposed observers to be implemented on UAV's. The critical issue in this domain comes from the fact that the translation velocity is classically known as non observable. A key contribution of the present work is to show that although the system linearized approximation is non-observable in the origin, the full nonlinear system may become observable under suitable sufficient conditions, thus allowing its estimation.

Motivated by the above issues, in this note we describe a novel observation strategy that solves the problem of the speed estimation of an Unmanned Aerial Vehicle, when the translational acceleration (in the local reference frame), the angles and the angular speeds (in the global reference frame) are available for measurement. We focus our analysis on a prototype drone - a 4 rotors helicopter robot shown in Fig. 1 - produced to operate in an urban environment at the Laboratoire IBISC - CNRS, Universite d'Evry, which is not equipped with GPS related devices (see [25]). We provide a global exponential solution to this open problem in the light of classical adaptive estimation theory when exact measurements are available. A modified estimator is presented to enhance robustness in velocity estimation in the case of noisy acceleration measurements. The simulation results obtained on the model of the real drone -even in the presence of noise- provide an additional algorithm validation test. The proposed scheme is relatively simple and this is a key issue since the algorithm has to be implemented in small embedded micro-controllers without heavy computation burden. 


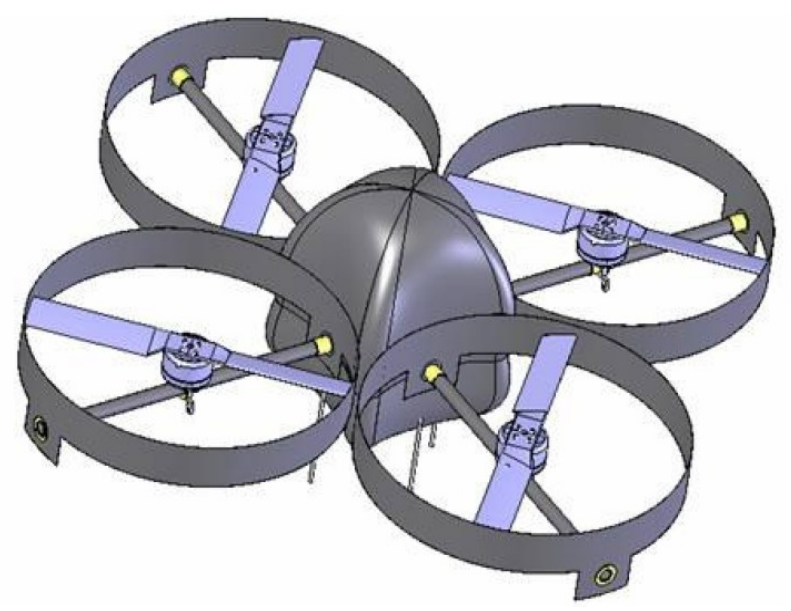

Figure 1: Prototype Drone

The results in this paper are inspired by two design principles. The first one is that by increasing the use of model based informations, it is possible to better exploit sensors estimations, which is obviously obtained via a much larger modeling effort and theoretical complexity. Second, by exploiting the reference frame changes of coordinates, there are new informations that may arise when some rather mild conditions of persistence of excitation (PE) are fulfilled. This property in turn is satisfied when the considered aerial vehicle has decoupled rotational and translational dynamics, since we aim at constructing an observer which estimates translational states based on rotational information. These design principles can be implemented on any vehicle such that rotational dynamics can be stabilized independently on the estimation of translational velocities, like helicopters and Vertical Take Off and Landing (VTOL) airplanes.

The nonlinear observability study introduced in this paper finds a natural complement in the design of an Extended Kalman Filter (EKF) for the considered UAV, which is used in the simulation section to illustrate and compare the behavior of the proposed adaptive observer. The tests performed show that the adaptive observer has a better performance in the case of internal and external disturbances like wind, unmodelled dynamics and measurement noise. Furthermore the proposed observers were designed based on adaptive theory and as consequence, present a self-tuning behavior much easier to set than the EKF.

The paper is organized as follows: in Section 2 we describe the drone model and formulate the problem addressed in this paper, analyzing the observability property of the system. This is guaranteed introducing a suitable PE assumption which is used in Section 3 to construct the observation algorithms and to prove that the estimation error converges exponentially to zero. In Section 4 we report some simulations that illustrate the observer performance and stress the influence of some parameters on the observer behavior. Finally in Section 5 we conclude the note with few remarks along with an outline of our future research on this topic.

\section{Problem formulation}

In this section we describe (see [2]) the UAV dynamic model equations and state the problem that we solve in the next sections. It is important to remark that the considered drone is a modified quadrotor that presents the interesting feature of swinging two of its motors, similar to a Vertical Take Off and Landing (VTOL) airplane, that is represented as two additional inputs than the standard quadrotors. This feature is not used in the present work, since it does not address the UAV's control, but it could be an interesting feature in a different setting.

Let $\eta_{1} \triangleq[x, y, z]^{T}$ be the UAV position vector represented in the global reference frame, let $\eta_{2} \triangleq$ $\left[\begin{array}{lll}\phi, & \theta, & \psi\end{array}\right]^{T}$ be the Euler angles vector represented in the global reference frame (roll pitch and yaw

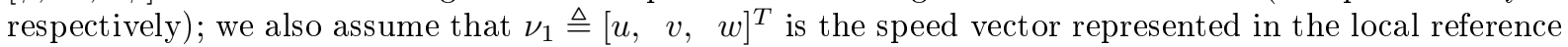
frame (surge, sway and heave respectively) and $\nu_{2} \triangleq\left[\begin{array}{lll}p, & q, & r\end{array}\right]^{T}$ is the angular speed vector represented in 
the local reference frame. The UAV model in state space form collects the set of the first order differential equations expressing $\dot{\eta}_{1}, \dot{\eta}_{2}, \dot{\nu}_{1}, \dot{\nu}_{2}$, as a function of $\eta_{1}, \eta_{2}, \nu_{1}, \nu_{2}$. To be more specific, the linear velocity in the fixed reference frame $\dot{\eta}_{1}=(\dot{x}, \dot{y}, \dot{z})^{T}$ can be expressed as a function of $\eta_{2}, \nu_{1}$, via the set of differential equalities

$$
\begin{aligned}
\dot{x}= & \cos (\theta) \cos (\psi) u \\
& +(\sin (\phi) \sin (\theta) \cos (\psi)-\cos (\phi) \sin (\psi)) v \\
& +(\cos (\phi) \sin (\theta) \cos (\psi)+\sin (\phi) \sin (\psi)) w \\
\dot{y}= & \cos (\theta) \sin (\psi) u \\
& +(\sin (\phi) \sin (\theta) \sin (\psi)+\cos (\phi) \cos (\psi)) v \\
& +(\cos (\phi) \sin (\theta) \sin (\psi)-\sin (\phi) \cos (\psi)) w \\
\dot{z}= & -\sin (\theta) u+\sin (\phi) \cos (\theta) v+\cos (\phi) \cos (\theta) w
\end{aligned}
$$

The time derivatives of the Euler angles $\dot{\eta}_{2}=(\dot{\phi}, \dot{\theta}, \dot{\psi})$ satisfy the following equations :

$$
\begin{aligned}
\dot{\phi} & =p+(\sin (\phi) q+\cos (\phi) r) \tan (\theta) \\
\dot{\theta} & =\cos (\phi) q-\sin (\phi) r \\
\dot{\psi} & =(\sin (\phi) q+\cos (\phi) r) \cos (\theta)^{-1}
\end{aligned}
$$

We assume that the UAV four rotors turn respectively at speed $\omega_{1}, \omega_{2}, \omega_{3}$ and $\omega_{4}$. Support of rotors 1 and 3 can also swivel around their pitch axes (angles $\varsigma_{1}$ and $\varsigma_{3}$ represent the orientation of each support). Theses quantities represent the system's control input functions and are related to the time derivatives of the speed vectors, respectively $\dot{\nu}_{1}=\left[\begin{array}{lll}\dot{u} & \dot{v} & \dot{w}\end{array}\right]^{T}$ and $\dot{\nu}_{2}=\left[\begin{array}{lll}\dot{p} & \dot{q} & \dot{r}\end{array}\right]^{T}$ which are accelerations in the local reference frame and comply with the mechanical equations of the UAV. In particular, we have

$$
\begin{aligned}
\dot{u}= & (-q w+r v-g \sin (\theta))-\frac{k_{T}}{m}\left(\omega_{1}^{2} \sin \left(\varsigma_{1}\right)+\omega_{3}^{2} \sin \left(\varsigma_{3}\right)\right) \\
\dot{v}= & (-r u+p w+g \sin (\phi) \cos (\theta)) \\
\dot{w}= & (-p v+q u+g \cos (\phi) \cos (\theta)) \\
& -\frac{k_{T}}{m}\left(\omega_{1}^{2} \cos \left(\varsigma_{1}\right)+\omega_{2}^{2}+\omega_{3}^{2} \cos \left(\varsigma_{3}\right)+\omega_{4}^{2}\right)
\end{aligned}
$$

where $g$ is the gravity acceleration constant, $m$ is the drone mass, $k_{T}$ is a constant relating the UAV rotor speeds and resulting thrust. In the angular setting we obtain

$$
\begin{aligned}
I_{x x} \dot{p}= & -\left(I_{z z}-I_{y y}\right) r q+l_{b} k_{T}\left(\omega_{1}^{2} \cos \left(\varsigma_{1}\right)-\omega_{3}^{2} \cos \left(\varsigma_{3}\right)\right) \\
& -q I_{r}\left(\omega_{1} \cos \left(\varsigma_{1}\right)+\omega_{2}+\omega_{3} \cos \left(\varsigma_{3}\right)+\omega_{4}\right) \\
& -k_{M}\left(\omega_{1}^{2} \sin \left(\varsigma_{1}\right)+\omega_{3}^{2} \sin \left(\varsigma_{3}\right)\right) \\
I_{y y} \dot{q}= & -\left(I_{x x}-I_{z z}\right) p r-r I_{r}\left(\omega_{1} \sin \left(\varsigma_{1}\right)+\omega_{3} \sin \left(\varsigma_{3}\right)\right) \\
& +p I_{r}\left(\omega_{1} \cos \left(\varsigma_{1}\right)+\omega_{2}+\omega_{3} \cos \left(\varsigma_{3}\right)+\omega_{4}\right) \\
& +l_{b} k_{T}\left(\omega_{2}^{2}-\omega_{4}^{2}\right)+u_{G} k_{T}\left(-\omega_{1}^{2} \sin \left(\varsigma_{1}\right)+\omega_{3}^{2} \sin \left(\varsigma_{3}\right)\right) \\
I_{z z} \dot{r}= & -\left(I_{y y}-I_{x x}\right) p q-k_{b}\left(\omega_{1}^{2} \sin \left(\varsigma_{1}\right)-\omega_{3}^{2} \sin \left(\varsigma_{3}\right)\right) \\
& +k_{M}\left(\omega_{1}^{2} \cos \left(\varsigma_{1}\right)-\omega_{2}^{2}+\omega_{3}^{2} \cos \left(\varsigma_{3}\right)-\omega_{4}^{2}\right) \\
& +q I_{r}\left(\omega_{1} \sin \left(\varsigma_{1}\right)+\omega_{3} \sin \left(\varsigma_{3}\right)\right)
\end{aligned}
$$

where $I_{x x}, I_{y y}, I_{z z}$ are the inertia moments, $k_{M}$ is the constant relating rotor speeds and torque, $l_{b}$ is the length of each drone's arm, and $I_{r}$ is the rotor's inertia moment constant.

In this paper we assume that the following quantities are available for measurement: orientation (Euler Angles in the global reference frame), angular velocities (in the local reference frame) and translational acceleration (in the local reference frame) of the vehicle. The coefficients $k_{T}$ and $k_{M}$ as well as different parameters of the model are experimentally identified. In this context ${ }^{1}$, defining the vectors

$$
\left\{\begin{array}{l}
X=[\phi, \theta, \psi, p, q, r, u, v, w]^{T} \\
y_{m}=[\phi, \theta, \psi, p, q, r, \dot{u}, \dot{v}, \dot{w}]^{T} \\
\Omega=\left[\omega_{1}, \omega_{2}, \omega_{3}, \omega_{4}, \varsigma_{1}, \varsigma_{3}\right]^{T}
\end{array}\right.
$$

\footnotetext{
${ }^{1}$ We will not consider position estimation. For this reason, the state vector does not include it
} 
equations (2)-(4) can be written in a compact notation as

$$
\left\{\begin{array}{l}
\dot{X}=f(X, \Omega), \quad X \in \mathbb{R}^{9} \\
y_{m}=h(X, \Omega), \quad y \in \mathbb{R}^{6}
\end{array}\right.
$$

where $f: \mathbb{R}^{9} \times \mathbb{R}^{6} \rightarrow \mathbb{R}^{9}$ is a smooth function depending on the state and the control input $\Omega$, and $h(X, \Omega)$ is obtained by combining the second equality in (5) along with (3), expressing $\dot{u}, \dot{v}, \dot{w}$ as functions of $X$ and $\Omega$.

The paper objective may then be stated as: given system (6), design an observation strategy yielding exponentially converging estimates of the state $X$ from the measurable output $y_{m}$, to be eventually included in any control strategy of system (6).

In practical terms, the observation algorithm focuses on the estimation of the unmeasurable state variables $\nu_{1}$, based on the measurable variables $\eta_{2}, \dot{\nu}_{1}$ and $\nu_{2}$ given by the standard sensors embedded in the drone. We remark that a necessary condition for the synthesis of any observer is the local observability property to be verified. To this purpose, we recall the following definition.

Definition 1 (see [13], [22]). Let $G$ denote the set of all finite linear combinations of the Lie derivatives of $h_{1}, \ldots, h_{p}$ with respect to $f$ for various values of $u=$ constant. Let $d G$ denote the set of all their gradients. If we can find $n$ linearly independent vectors within $d G$, then the system is locally observable.

The observability matrix $\mathcal{O} \triangleq d G$ is given by :

$$
\mathcal{O}=\left(\begin{array}{c}
L_{f}^{0} h \\
\cdots \\
L_{f}^{p-1} h
\end{array}\right)
$$

The system is locally observable if $\mathcal{O}$ has full rank. Thus in the case we consider in this paper, the estimation strategy has to be coupled with a tracking control algorithm driving the angular speeds $[p, q, r]^{T}$ to bounded and non vanishing reference trajectories $\left[p_{r}(t), q_{r}(t), r_{r}(t)\right]^{T}$ that guarantee the rank of matrix $\mathcal{O}$ to be different from zero. To be more specific, let $\Phi(t)$ be the matrix defined as

$$
\Phi(t)=\left[\begin{array}{ccc}
0 & r & -q \\
-r & 0 & p \\
q & -p & 0
\end{array}\right]
$$

In this paper we assume $\Phi(t)$ is persistently exciting (PE), as formally stated below.

Hypothesis 1 The matrix $\Phi(t)$ is bounded and persistently exciting $(P E)$, i.e. there are positive integers $T^{*}, K_{1}^{*}$ and $K_{2}^{*}$ such that

$$
K_{1}^{*} I \geq \int_{t}^{t+T^{*}} \Phi(\tau)^{T} \Phi(\tau) d \tau \geq K_{2}^{*} I \quad \text { for all } t \in \mathbb{R}
$$

Claim 1 Hypothesis (H1) is satisfied for any speed vector represented in the local reference frame $\nu_{2}=$ $[p, q, r]^{T}$, which is bounded and PE, i.e. such that

$$
c_{1}^{*} I \geq \int_{t}^{t+T^{*}} \nu_{2}(\tau)^{T} \nu_{2}(\tau) d \tau \geq c_{2}^{*} I \quad \text { for all } t \in \mathbb{R}
$$

for suitable positive integers $T^{*}, c_{1}^{*}$ and $c_{2}^{*}$.

Proof. To prove the Claim we show that any $\nu_{2}(t)$ which does not comply with (8) does not comply with (9) also. In fact, if $\Phi(t)$ is not PE, then there exists a nonzero vector $s=\left[s_{1}, s_{2}, s_{3}\right]^{T}$ such that $\lim _{t \rightarrow \infty}\|\Phi(t) s\|^{2}=0$. By expanding the term $\|\Phi(t) s\|^{2}$, recalling (7), we deduce that the limit identity above implies

$$
s_{3} \lim _{t \rightarrow \infty} q(t)=s_{2} \lim _{t \rightarrow \infty} r(t) ; \quad s_{3} \lim _{t \rightarrow \infty} p(t)=s_{1} \lim _{t \rightarrow \infty} r(t) ; \quad s_{2} \lim _{t \rightarrow \infty} p(t)=s_{1} \lim _{t \rightarrow \infty} q(t)
$$


Property (10) can be expressed concisely as $\nu_{2}(t)=\operatorname{Tr}(t) V+\epsilon(t)$ where $V \in \mathbb{R}^{3}, V \neq 0$ is any non zero vector, $\operatorname{Tr}(t)$ is any scalar time function and $\epsilon(t)$ is any vector function such that $\lim _{t \rightarrow \infty} \epsilon(t)=0$. It is straightforward to show that any $\nu_{2}(t)$ in the form above does not satisfy (9), and this proves the Claim.

Remark 1 By virtue of Claim 1, Hypothesis 1 is guaranteed for any angular speed vector $\nu_{2}(t)$ that spans periodically a 3-dimensional surface, for instance a non-vanishing periodic orbit. Besides, the nature of the vehicle provides vibrations in real applications, that may already give enough persistence of excitation which relyes on angular speeds (oscillation frequency) rather than angles (oscillation amplitudes).

Remark 2 The necessity of Hypothesis 1 clarifies that our estimation strategy can be implemented on that set of aerial vehicles satisfying PE condition. All helicopters and quadrotors fulfill this requirement because their rotational and translational dynamics are decoupled and the rotational states are available for measurement with standard sensors (IMU, infrared sensors, magnetometers). For this reason, it is possible to track a persistently exciting reference independently of estimation results. These could then be used in an outer loop that may be used to stabilize the translational dynamics.

\section{Observer Design}

In this section we describe the estimation strategy following the techniques presented in [19] and [20], as well as an outline of its stability proof. Two different algorithms that estimate the linear velocity of the system described by equations (2) - (4) are presented. The first algorithm considers the ideal case with no noise on the measurement outputs while the second takes into account additive measurement noise on the translational accelerations.

The measured output can be expressed as

$$
y_{m} \triangleq\left[y_{1}, y_{2}\right]^{T}=[\underbrace{\phi, \theta, \psi, p, q, r}_{y_{1}}, \underbrace{\dot{u}, \dot{v}, \dot{w}}_{y_{2}}]^{T}
$$

where the vector $y_{2}=\dot{\nu}_{1}=[\dot{u}, \dot{v}, \dot{w}]^{T}$ is available for measurement, so that equation (3) is re-written as

$$
\begin{aligned}
\dot{\nu}_{1}(t) & =\Phi(t) \nu_{1}(t)+\Lambda(t) \\
y_{2} & =\dot{\nu}_{1}
\end{aligned}
$$

with the matrix $\Phi$ as in (7) and the vector $\Lambda$ defined as

$$
\Lambda(t)=\left[\begin{array}{c}
-g \sin (\theta)-\frac{k_{T}}{m}\left(\omega_{1}^{2} \sin \left(\varsigma_{1}\right)+\omega_{3}^{2} \sin \left(\varsigma_{3}\right)\right) \\
g \sin (\phi) \cos (\theta) \\
g \cos (\phi) \cos (\theta)-\frac{k_{T}}{m}\left(\omega_{1}^{2} \cos \left(\varsigma_{1}\right)+\omega_{2}^{2}+\omega_{3}^{2} \cos \left(\varsigma_{3}\right)+\omega_{4}^{2}\right)
\end{array}\right]
$$

In the following we design a reduced-order observer which estimates the translational velocity of the UAV recalling that, in order to guarantee that $p(t), q(t)$ and $r(t)$ are different from zero, the control inputs have to be chosen such that the system oscillates around the equilibrium point.

\subsection{Basic algorithm}

In this section we construct an algorithm to estimate the time function $\nu_{1}(t)$ assuming, by virtue of (11), that the quantity $Y_{1} \triangleq\left[\dot{\nu}_{1}(t)-\Lambda(t)\right]=\Phi(t) \nu_{1}(t)$ is measurable, recalling that both the time derivative of the function to be estimated $y_{2}=\dot{\nu}_{1}(t)$ and the regression matrix $\Phi(t)$ are available for measurement. We remark that many adaptive estimation techniques (see [20]) are shown to guarantee exponential function estimation when the time derivative of the regression matrix is also available for measurement, thus the first step in the estimation strategy is to replace the regression identity $Y_{1}=\Phi(t) \nu_{1}(t)$ with another identity $Y_{2} \triangleq M(t) \nu_{1}(t)$, where $M(t)$ is a matrix (to be defined) whose time derivative $\dot{M}$ is available for measurement. To this purpose, let $M(t) \in \mathbb{R}^{3} \times \mathbb{R}^{3}$ be the state of the filter

$$
\dot{M}=-\alpha M+\Phi(t)
$$

with arbitrary initial condition $M(0) \in \mathbb{R}^{3} \times \mathbb{R}^{3}$, where $\alpha \in \mathbb{R}^{+}$is a positive tuning parameter chosen by the designer. The matrix $M(t)$ is also PE, as stated below. 
Claim 2 The matrix $M$ is persistently exciting, i.e. there exist positive integers $K_{M 1}^{*}$ and $K_{M 2}^{*}$ such that

$$
K_{M 1}^{*} I \geq \int_{t}^{t+T^{*}} M(\tau)^{T} M(\tau) d \tau \geq K_{M 2}^{*} I \quad \text { for all } t \in \mathbb{R}
$$

where $T^{*}$ is the positive real defined in Hypothesis $H 1$.

Proof. By contradiction, assume that there exists a vector $s \in \mathbb{R}^{3}$ with $\|s\|=1$ such that $\lim _{t \rightarrow \infty} M(t) s=$ 0 . Then by setting $\Delta M(t)=M\left(t+T^{*}\right) s-M(t) s$ we have that $\lim _{t \rightarrow \infty} \Delta M(t)=0$. Recalling that by virtue of (12) $\Delta M(t)=-\alpha \int_{t}^{t+T^{*}} M(\tau) s d \tau+\int_{t}^{t+T^{*}} \Phi(\tau) s d \tau$, we deduce that $\lim _{t \rightarrow \infty} \int_{t}^{t+T^{*}} \Phi(\tau) s d \tau=0$, contradicting hypothesis (H1).

Define the system

$$
\dot{\rho}=-\alpha \rho+\dot{\nu}_{1}-\Lambda(t)+M \dot{\nu}_{1}=-\alpha \rho+y_{2}-\Lambda(t)+M y_{2}
$$

with state $\rho \in \mathbb{R}^{3}$, arbitrary initial condition $\rho(0) \in \mathbb{R}^{3}$. The task of (12) and (14) is to obtain a measurable estimate $\rho$ of the vector $Y_{2}=M \nu_{1}$ with $M$ measurable along with its time derivative. In fact, by setting $\sigma \triangleq \rho-M \nu_{1}$ we have

$$
\dot{\sigma}=-\alpha\left(\rho-M \nu_{1}\right)=-\alpha \sigma
$$

which implies that $\sigma$ exponentially converges to zero.

Consider the filter

$$
\dot{Q}=-\beta Q+M^{T} M
$$

with state $Q \in \mathbb{R}^{3} \times \mathbb{R}^{3}$ and any initial condition such that $Q(0)>0$, where $\beta \in \mathbb{R}^{+}$is a positive tuning parameter chosen by the designer. From (16) by using the arguments in [20] we demonstrate the following result.

Claim 3 Assume that $Q(0)>0$; then the matrix $Q(t)$ is bounded and positive definite, i.e. there exists a positive constant $c_{2}$ such that $Q(t)>c_{2} I$ for all $t \geq 0$.

Proof. Each entry of the matrix $Q(t)$ is the state of a linear asymptotically stable filter driven by a bounded input, so that $Q(t)$ is bounded. In order to prove that $Q(t)$ is positive definite, from (16) we have that

$$
Q(t)=e^{-\beta t} Q(0)+\int_{0}^{t} e^{-\beta(t-\tau)} M(\tau)^{T} M(\tau) d \tau
$$

Recall that $e^{-\beta t} Q(0)>0$ and $\int_{0}^{t} e^{-\beta(t-\tau)} M(\tau)^{T} M(\tau) d \tau \geq 0$ for all $t>0$, thus $Q(t) \geq e^{-\beta T^{*}} Q(0)$ for all $0 \leq t \leq T^{*}$; however from (16) we also infer that $Q\left(T^{*}\right) \geq \int_{0}^{T^{*}} e^{-\beta\left(T^{*}-\tau\right)} M(\tau)^{T} M(\tau) d \tau \geq$ $e^{-\beta T^{*}} \int_{0}^{T^{*}} M(\tau)^{T^{*}} M(\tau) d \tau$, that by virtue of (13) in Claim 2 yields $Q\left(T^{*}\right) \geq e^{-\beta T^{*}} K_{M 2}^{*} I$. By repeating iteratively these arguments we have that $Q(t) \geq e^{-2 \beta T^{*}} K_{M 2}^{*} I$ in each interval $i T^{*} \leq t \leq(i+1) T^{*}$ with $i=1,2 \ldots \infty$, concluding that $Q(t) \geq c_{2} I$ where $c_{2}=\min \left\{e^{-\beta T^{*}} Q(0), e^{-2 \beta T^{*}} K_{M 2}^{*}\right\}$.

Thus the idea behind the observation strategy in this note is to seek an estimate

$$
\hat{\nu}_{1}=\left[\begin{array}{c}
\hat{u} \\
\hat{v} \\
\hat{w}
\end{array}\right]
$$

of $\nu_{1}$ such that by setting the estimation error variable $\tilde{\nu}_{1} \triangleq \nu_{1}-\hat{\nu}_{1}$, its time derivative can be written in the form

$$
\dot{\tilde{\nu}}_{1}=\gamma\left(-Q \tilde{\nu}_{1}+\chi\right)
$$

where $\gamma \in \mathbb{R}^{+}$is a positive tuning parameter to be chosen by the designer and $\chi$ is a quantity to be defined within the synthesis process. Notice that $\chi$ must not be chosen necessarily as a measurable quantity, rather it has to converge exponentially to zero. If this is the case, then the positive definiteness of $Q(t)$ implies that $\lim _{t \rightarrow \infty} \tilde{\nu}_{1}(t)=0$ exponentially. Since $\hat{\nu}_{1}=\nu_{1}-\tilde{\nu}_{1}$, the computation of $\dot{\hat{\nu}}_{1}$ along with (17) yields

$$
\dot{\hat{\nu}}_{1}=\gamma\left(-Q \hat{\nu}_{1}+Q \nu_{1}-\chi\right)+y_{2}
$$


$>$ From the last expression we infer that $\chi$ has to include the non measurable term $Q \nu_{1}$ for the dynamics $\dot{\hat{\nu}}_{1}$ to be implementable. Notice that $d\left(Q \nu_{1}\right) / d t=-\beta\left(Q \nu_{1}\right)+M^{T} M \nu_{1}+Q y_{2} ;$ this circumstance suggests that $\chi$ has to be set equal to $Q \nu_{1}$ plus the states of some stable linear filters with eigenvalue $-\beta$ fed by terms compensating $M^{T} M \nu_{1}$ and $Q y_{2}$. In particular, defining these stable filters as

$$
\begin{gathered}
\dot{\delta}=-\beta \delta+\rho \triangleq-\beta \delta+\sigma+M \nu_{1} \\
\dot{\xi}=-\beta \xi+Q y_{2}+[\alpha M-\Phi(t)]^{T} \delta \triangleq-\beta \xi+Q y_{2}-\dot{M}^{T} \delta
\end{gathered}
$$

with vector states $\delta \in \mathbb{R}^{3}, \xi \in \mathbb{R}^{3}$, arbitrary initial conditions $\delta(0) \in \mathbb{R}^{3} \xi(0) \in \mathbb{R}^{3}$ and setting

$$
\chi \triangleq Q \nu_{1}-M^{T} \delta-\xi
$$

by taking into account (19), (20) we obtain

$$
\dot{\chi}=-\beta \chi-M^{T} \sigma
$$

$>$ From (22) we infer that $\chi$ is the state of a stable filter driven by the exponentially vanishing input $\sigma$, so that $\lim _{t \rightarrow \infty} \chi(t)=0$ exponentially, which implies that $\lim _{t \rightarrow \infty} \tilde{\nu}_{1}(t)=0$ exponentially. Equality (21) along with (18) yields the dynamics for $\hat{\nu}_{1}$, the estimate of $\nu_{1}$, given by

$$
\dot{\hat{\nu}}_{1}=\gamma\left(-Q \hat{\nu}_{1}+M^{T} \delta+\xi\right)+y_{2} \text {. }
$$

Previous arguments can be summarized as follows.

Proposition 1 Consider the UAV model described by (2)-(4). Assume that the variables $\eta_{2}, \nu_{2}, \dot{\nu}_{1}$ are available for measurement and that Hypothesis 1 holds. Then for all $\alpha \in \mathbb{R}^{+}, \beta \in \mathbb{R}^{+}, \gamma \in \mathbb{R}^{+}$, the dynamic observer described by (12), (14), (16), (19), (20) and (23) with state $M(t) \in \mathbb{R}^{3} \times \mathbb{R}^{3}$, $Q(t) \in \mathbb{R}^{3} \times \mathbb{R}^{3}, \rho(t) \in \mathbb{R}^{3}, \delta(t) \in \mathbb{R}^{3}, \xi(t) \in \mathbb{R}^{3}$ and $\hat{\nu}_{1}(t) \in \mathbb{R}^{3}$ is such that the vector $\nu_{1}-\hat{\nu}_{1}$ converges to zero globally exponentially for any initial conditions $M(0) \in \mathbb{R}^{3} \times \mathbb{R}^{3}, Q(0) \in \mathbb{R}^{3} \times \mathbb{R}^{3}, \rho(0) \in \mathbb{R}^{3}$, $\delta(0) \in \mathbb{R}^{3}, \xi(0) \in \mathbb{R}^{3} \hat{\nu}_{1}(0) \in \mathbb{R}^{3}$ with $Q(0)>0$.

Proof. The result has been already outlined in the previous arguments; a rigorous demonstration follows from standard Lyapunov theory. In particular, consider the class of Lyapunov functions

$$
V=\frac{1}{2} \kappa_{1}\left\|\tilde{\nu}_{1}\right\|^{2}+\frac{1}{2} \kappa_{2}\|\chi\|^{2}+\frac{1}{2} \kappa_{3}\|\sigma\|^{2}
$$

where $\kappa_{1}, \kappa_{2}, \kappa_{3}$, are positive real numbers to be defined later. By computing the time derivative $\dot{V}$ and recalling (15), (22), (17) we obtain

$$
\begin{aligned}
\dot{V}= & -\alpha \kappa_{3}\|\sigma\|^{2}-\beta \kappa_{2}\|\chi\|^{2}-\kappa_{2} \chi^{T} M^{T} \tilde{z} \\
& -\gamma \kappa_{1} \tilde{\nu}_{1}^{T} Q \tilde{\nu}_{1}+\gamma \kappa_{1} \tilde{\nu}_{1}^{T} \chi .
\end{aligned}
$$

Notice that Hypothesis 1 implies that matrix $M(t)$ is also bounded and persistently exciting. From (16) by using the arguments in [20] it is shown that if $Q(0)>0$, then matrix $Q(t)$ is positive definite for all $t \geq 0$. Setting the positive constant $c_{1}=\sup _{t \in[0, \infty)}\|M(t)\|^{2}$, we deduce

$$
\begin{aligned}
\kappa_{2} \chi^{T} M^{T} \sigma & \leq \frac{\kappa_{2} \beta}{4}\|\chi\|^{2}+\frac{\kappa_{2}}{\beta} c_{1}^{2}\|\sigma\|^{2} \\
\gamma \chi^{T} \tilde{\nu}_{1} & \leq \frac{\gamma c_{2}}{2}\left\|\tilde{\nu}_{1}\right\|^{2}+\frac{\gamma}{2 c_{2}}\|\chi\|^{2}
\end{aligned}
$$

where $c_{2}$ is the bound defined in Claim 3. The substitution of the previous inequalities in (25) yields

$$
\begin{aligned}
\dot{V} \leq & -\left(\kappa_{3} \alpha-\frac{\kappa_{2}}{\beta} c_{1}^{2}\right)\|\sigma\|^{2}-\left(\kappa_{2} \beta-\frac{\kappa_{2} \beta}{4}+\frac{\kappa_{1} \gamma}{2 c_{2}}\right)\|\chi\|^{2} \\
& -\kappa_{1} \gamma\left(c_{2}-\frac{c_{2}}{2}\right)\left\|\tilde{\nu}_{1}\right\|^{2}
\end{aligned}
$$


By setting $\kappa_{1}=1, \kappa_{2}=\frac{2 \gamma}{\beta c_{2}}, \kappa_{3}=\frac{2 \kappa_{2} c_{1}^{2}}{\beta \alpha}=\frac{4 \gamma c_{1}^{2}}{\beta^{2} \alpha c_{2}}$, we finally obtain

$$
\dot{V} \leq-\left(\frac{\gamma c_{2}}{2}\right)\left\|\tilde{\nu}_{1}\right\|^{2}-\left(\frac{\gamma}{2 c_{2}}\right)\|\chi\|^{2}-\left(\frac{2 \gamma c_{1}^{2}}{\beta^{2} c_{2}}\right)\|\sigma\|^{2}
$$

Let $\lambda_{1}=\min \left\{\gamma c_{2}, \frac{\beta}{2}, \alpha\right\}$. Previous inequality implies that $\dot{V} \leq-\lambda_{1} V$, which guarantees the convergence of all errors exponentially to zero.

\subsection{Extension of the basic algorithm}

In this section, the former algorithm is modified in order to quantify the observer performance in case of measurement noise applied on the velocity and acceleration signals. We assume now that the noisy acceleration $a_{\mu}$ and the noisy velocity matrix $\Phi_{\mu}(t)$ to be available for measurement, given by

$$
a_{\mu}=\left[\begin{array}{c}
\dot{u}+\mu_{11}(t) \\
\dot{v}+\mu_{12}(t) \\
\dot{w}+\mu_{13}(t)
\end{array}\right] ; \quad \Phi_{\mu}(t)=\left[\begin{array}{ccc}
0 & r+\mu_{21}(t) & -q-\mu_{22}(t) \\
-r-\mu_{21}(t) & 0 & p+\mu_{23}(t) \\
q+\mu_{22}(t) & -p-\mu_{23}(t) & 0
\end{array}\right]
$$

where $\mu_{i j}(t), i=1,2, j=1,2,3$ are bounded measurement noises, i.e. are such that

$$
\left\|\mu_{i j}(t)\right\| \leq \mu_{M}, \text { for all } t>0, i=1,2, j=1,2,3,
$$

with $\mu_{M} \in \mathbb{R}^{+}$a suitable positive bound. In this framework, the measurement noises play the role of "small" non vanishing exogenous inputs affecting the estimation error dynamics. The observation strategy in this setting focuses on the synthesis of a "robust" observer, i.e. such that noise influence on estimation error is "small". To comply with this requirement, extra tuning parameters will be introduced in the observer design, which also aims at Input-to-State Stability property (see [14] for ISS definition): in practice, the observer estimation error is attracted inside an open ball whose radius depends on the measurement noise. To adapt ISS property to the design we consider in this note, we recall the following result, that is demonstrated via standard Lyapunov theory.

Lemma 1 Consider the system

$$
\dot{x}=A(t) x+B(t) u
$$

with state $x(t) \in \mathbb{R}^{n}$, arbitrary initial condition $x(0) \in \mathbb{R}^{n}$ and input $u(t) \in \mathbb{R}^{m}$. Assume that:

(i) there is a positive scalar parameter $\lambda_{m}$ such that $v^{T} A(t) v \leq-\lambda_{m}\|v\|^{2}$ for all $t \geq 0$ and for any $v \in \mathbb{R}^{n}$;

(ii) there is a positive scalar parameter $\beta_{M}$ such that $\|B(t)\|^{2}<\beta_{M}$ for all $t \geq 0$.

If there are suitable positive constants $k_{u}, u_{M}, \lambda_{u}$ such that $\|u(t)\|^{2} \leq k_{u} \exp \left(-\lambda_{u} t\right)+u_{M}^{2}$ i.e. $u(t)$ is exponentially attracted to a ball with radius $u_{M}$, then there are suitable positive constants $k_{1}, k_{2}, \lambda_{x}$ such that

$$
\|x(t)\|^{2} \leq k_{1} \exp \left(-\lambda_{x} t\right)+k_{2} u_{M}^{2}
$$

i.e. $x(t)$ is also exponentially attracted to a ball whose radius is proportional by a suitable factor $k_{2}$ to the bound $u_{M}$ of the input $u(t)$.

Proof. Consider the Lyapunov function $V(t)=\frac{1}{2}\|x(t)\|^{2}$. By computing its time derivative, we have

$$
\dot{V}(t)=x^{T} A(t) x+x^{T} B(t) u .
$$

By completing the squares, recalling (i), (ii), we obtain $x^{T} A(t) x \leq-\lambda_{m}\|x\|^{2}$ and $x^{T} B(t) u \leq$ $\frac{\lambda_{m}}{2}\|x(t)\|^{2}+\frac{\beta_{M}}{2 \lambda_{m}}\|u(t)\|^{2}$, which substituted into (29) and recalling the definition of $V$ yields

$$
\dot{V}(t) \leq-\lambda_{m} V(t)+\frac{\beta_{M}}{2 \lambda_{m}}\left[k_{u} \exp \left(-\lambda_{u} t\right)+u_{M}^{2}\right] .
$$

Set $\lambda^{*}=\min \left\{\frac{\lambda_{m}}{2}, \lambda_{u}\right\}$. Previous inequality implies that $V(t) \leq W(t)$, where $W(t)$ complies with the differential equality

$$
\dot{W}(t)=-\lambda_{m} W(t)+\frac{\beta_{M}}{2 \lambda_{m}}\left[k_{u} \exp \left(-\lambda^{*} t\right)+u_{M}^{2}\right]
$$


with initial condition $W(0)=V(0)=\frac{1}{2}\|x(0)\|^{2}$. The solution of $(30)$ is

$$
W(t)=W(0) \exp \left(-\lambda_{m} t\right)+\frac{\beta_{M}}{2 \lambda_{m}}\left[\frac{k_{u}}{\lambda_{m}-\lambda^{*}}\left(e^{-\lambda^{*} t}-e^{-\lambda_{m} t}\right)+\frac{u_{M}^{2}}{\lambda_{m}}\left(1-e^{-\lambda_{m} t}\right)\right] .
$$

Neglecting the negative terms in previous equality, we have

$$
W(t) \leq W(0) \exp \left(-\lambda_{m} t\right)+\frac{\beta_{M}}{2 \lambda_{m}}\left[\frac{k_{u}}{\lambda_{m}-\lambda^{*}} e^{-\lambda^{*} t}+\frac{u_{M}^{2}}{\lambda_{m}}\right]
$$

recalling that $\frac{1}{2}\|x(t)\|^{2}=V(t) \leq W(t)$ and $W(0) \exp \left(-\lambda_{m} t\right) \leq \frac{1}{2}\|x(0)\|^{2} \exp \left(-\lambda^{*} t\right)$, we deduce that (28) is verified by setting $\lambda_{x}=\lambda^{*}, k_{1}=\frac{\beta_{M} k_{u}}{\lambda_{m}\left(\lambda_{m}-\lambda^{*}\right)}+\|x(0)\|^{2}$ and $k_{2}=\frac{\beta_{M}}{\lambda_{m}^{2}}$.

The first step in the estimation strategy is the definition of the filters with states $M_{\mu} \in \mathbb{R}^{3} \times \mathbb{R}^{3}$, $\rho_{\mu} \in \mathbb{R}^{3}$, whose role is the same of filters (12), (14) in previous section:

$$
\begin{gathered}
\dot{M}_{\mu}=-\left(\alpha+\frac{k}{4}\right) M_{\mu}+\Phi_{\mu}(t) \\
\dot{\rho}_{\mu}=-\left(\alpha+\frac{k}{4}\right) \rho_{\mu}+a_{\mu}-\Lambda(t)+M_{\mu} a_{\mu}
\end{gathered}
$$

with arbitrary initial conditions, where $k \in \mathbb{R}^{+}, \alpha \in \mathbb{R}^{+}$, are tuning parameters chosen by the designer. By setting $\sigma_{\mu}=\rho_{\mu}-M_{\mu} \nu_{1}$ and $\mu_{1}(t)=\left[\mu_{11}(t), \mu_{12}(t), \mu_{13}(t)\right] \in \mathbb{R}^{3} \mu_{2}=\left[\mu_{21}(t), \mu_{22}(t), \mu_{23}(t)\right]^{T} \in \mathbb{R}^{3}$ and

$$
N_{2}(t)=\left[\begin{array}{ccc}
-v(t) & w(t) & 0 \\
u(t) & 0 & -w(t) \\
0 & -u(t) & v(t)
\end{array}\right]
$$

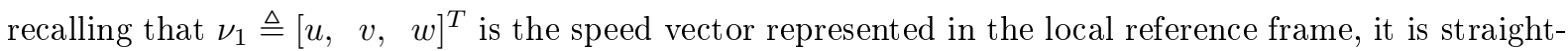
forward to verify that

$$
\dot{\sigma}_{\mu}=-\left(\alpha+\frac{k}{4}\right) \sigma_{\mu}+\left(M_{\mu}+I\right) \mu_{1}+N_{2} \mu_{2} .
$$

It is shown in the following that the dynamics of $\sigma_{\mu}(t)$ in (33) comply with the hypotheses of Lemma 1, i.e. $\sigma_{\mu}(t)$ is exponentially attracted inside a ball whose radius depends on the bounds of $\mu_{1}(t)$ and $\mu_{2}(t)$. Consider the filter with matrix state $Q_{\mu} \in \mathbb{R}^{3} \times \mathbb{R}^{3}$, with initial condition such that $\left|Q_{\mu}(0)\right|>0$, defined as:

$$
\dot{Q}_{\mu}=-\beta Q_{\mu}-\frac{k}{4} Q_{\mu} Q_{\mu}^{T} Q_{\mu}+M_{\mu}^{T} M_{\mu}
$$

where $\beta \in \mathbb{R}^{+}$is a tuning parameter chosen by the designer. The filter (34) is a generalization of system (16); by following arguments similar to the ones of previous section we show a result that generalizes Claim 3.

Claim 4 Assume that $Q_{\mu}(0)>0$; then the matrix $Q_{\mu}(t)$ is bounded and positive definite, i.e. $Q_{\mu}(t)>0$ for all $t \geq 0$.

Proof. First, recall that $Q_{\mu}$ is bounded. In fact, set $x_{\mu}=Q_{\mu} s$ where $s \in \mathbb{R}^{3}$ is any vector such that $\|s\|=1$. The computation of the time derivative of $x_{\mu}=Q_{\mu} s$, by virtue of (34) yields

$$
\dot{x}_{\mu}=-\left(\beta I+\frac{k}{4} Q_{\mu} Q_{\mu}^{T}\right) x_{\mu}+M_{\mu}^{T} M_{\mu} s
$$

$>$ From (35) we infer that $x_{\mu}$ complies with Lemma 1 with $\left[\beta I+\frac{k}{4} Q_{\mu}(t) Q_{\mu}^{T}(t)\right]$ in place of $A(t)$ and $\beta$ in place of $\lambda_{m}$, with $M_{\mu}^{T}(t) M_{\mu}(t)$ in place of $B(t)$ and $s \in \mathbb{R}^{3}$ in place of $u(t)$. Thus $x_{\mu}=Q_{\mu} s$ is exponentially attracted inside a ball whose radius depends on $\sup _{t \in[0, \infty)}\left\|M_{\mu}^{T}(t) M_{\mu}(t)\right\|$, so that (being $s \in \mathbb{R}^{3}$ arbitrary), we conclude that $Q_{\mu}$ is bounded; in particular, there exists $c_{Q} \in \mathbb{R}^{+}$such that

$$
\sup _{t \in[0, \infty)}\left\|Q_{\mu}(t)\right\| \leq c_{Q}
$$


In order to prove that $Q_{\mu}$ is positive definite, set $\eta_{\mu}=s^{T} Q_{\mu} s$, where $s \in \mathbb{R}^{3}$ is any vector such that $\|s\|=1$. The computation of the time derivative of $\eta_{\mu}$, by virtue of (34) along with (36) yields

$$
d \eta_{\mu} / d t \geq-\beta^{*} \eta_{\mu}+\left\|M_{\mu} s\right\|^{2}
$$

where $\beta^{*}=\beta+\frac{k}{4} c_{Q}^{2}$. From the inequality above we infer that

$$
\eta_{\mu}(t) \geq e^{-\beta^{*} t} \eta_{\mu}(0)+\int_{0}^{t} e^{-\beta^{*}(t-\tau)}\left\|M_{\mu}(\tau) s\right\|^{2} d \tau
$$

Recall that $e^{-\beta^{*} t} \eta_{\mu}(0)>0$ and $\int_{0}^{t} e^{-\beta^{*}(t-\tau)}\left\|M_{\mu}(\tau) s\right\|^{2} d \tau \geq 0$ for all $t>0$, thus $\eta_{\mu}(t) \geq e^{-\beta^{*} t} \eta_{\mu}(0)$ for all $0 \leq t \leq T^{*}$, where $T^{*}$ is the positive real defined in Hypothesis H1. From (16) we have that $\eta_{\mu}\left(T^{*}\right) \geq$ $\int_{0}^{T^{*}} e^{-\beta^{*}\left(T^{*}-\tau\right)}\left\|M_{\mu}(\tau) s\right\|^{2} d \tau \geq e^{-\beta^{*} T^{*}} \int_{0}^{T^{*}}\left\|M_{\mu}(\tau) s\right\|^{2} d \tau$. Remark that by following the same arguments in Claim 2 we deduce that $M_{\mu}$ is P.E., in particular there exists a suitable positive bound $K_{\mu 2}^{*}$ such that $\int_{0}^{T^{*}}\left\|M_{\mu}(\tau) s\right\|^{2} d \tau \geq K_{\mu 2}^{*}$, which implies $\eta_{\mu}\left(T^{*}\right) \geq e^{-\beta^{*} T^{*}} K_{\mu 2}^{*}$. By repeating iteratively these arguments we have that $\eta_{\mu}(t) \geq e^{-2 \beta^{*} T^{*}} K_{\mu 2}^{*}$ in each interval $i T^{*} \leq t \leq(i+1) T^{*}$ with $i=1,2 \ldots \infty$, so that $\eta_{\mu}(t) \geq q_{\mu}$, where $q_{\mu}=\min \left\{e^{-T^{*}} \eta_{\mu}(0), e^{-2 T^{*}} K_{\mu 2}^{*}\right\}$ and we conclude that $\left\|Q_{\mu}(t)\right\| \geq q_{\mu}$.

Consider the two filters with states $\delta_{\mu} \in \mathbb{R}^{3}$ and $\xi_{\mu} \in \mathbb{R}^{3}$, whose task is analogous to the one of (19), (20) respectively in previous section,

$$
\begin{gathered}
\dot{\delta}_{\mu}=-\beta \delta_{\mu}+\rho_{\mu} \\
\dot{\xi}_{\mu}=-\beta \xi_{\mu}-\frac{k}{4} Q_{\mu} Q_{\mu}^{T} \xi_{\mu}+Q_{\mu} a_{\mu}-\dot{M}_{\mu}{ }^{T} \delta_{\mu}-\frac{k}{4} Q_{\mu} Q_{\mu}^{T} M_{\mu}^{T} \delta_{\mu}
\end{gathered}
$$

with arbitrary initial conditions.

We introduce an estimate of the linear velocity

$$
\hat{\nu}_{1}=\left[\begin{array}{c}
\hat{u} \\
\hat{v} \\
\hat{w}
\end{array}\right]
$$

that satisfy the differential equations :

$$
\dot{\hat{\nu}}_{1}=\gamma\left(-Q_{\mu} \hat{\nu}_{1}+M_{\mu}^{T} \delta_{\mu}+\xi_{\mu}\right)+a_{\mu}
$$

with $\gamma \in \mathbb{R}^{+}$tuning parameter to be chosen by the designer.

Under Hypothesis 1, if the variables $\eta_{2}, \nu_{2}, a_{\mu}$ are available for measurement, the dynamic observer described by (31), (32), (34), (38), (39), and (40) with state $M_{\mu}(t) \in \mathbb{R}^{3} \times \mathbb{R}^{3}, \rho_{\mu}(t) \in \mathbb{R}^{3}, Q_{\mu}(t) \in$ $\mathbb{R}^{3} \times \mathbb{R}^{3}, \delta_{\mu}(t) \in \mathbb{R}^{3}, \xi_{\mu}(t) \in \mathbb{R}^{3}$ and $\hat{\nu}_{1}(t) \in \mathbb{R}^{3}$ is a generalization of the observer presented in Section 3.1. In fact, by setting the estimation error variables $\tilde{\nu}_{1} \triangleq \nu_{1}-\hat{\nu}_{1}$ and $\chi_{\mu} \triangleq Q \nu_{1}-M_{\mu}^{T} \delta_{\mu}-\xi_{\mu}$, we obtain:

$$
\begin{gathered}
\dot{\chi}_{\mu}=-\left(\beta+\frac{k}{4} Q_{\mu} Q_{\mu}^{T}\right) \chi_{\mu}-M_{\mu}^{T} \sigma_{\mu}-Q_{\mu} \mu_{1} \\
\dot{\tilde{\nu}}_{1}=\gamma\left(-Q_{\mu} \tilde{\nu}_{1}+\chi_{\mu}\right)-\mu_{1} .
\end{gathered}
$$

The iterative application of Lemma 1 to the systems (33), (41) and (42) leads to the demonstration of the convergence properties of the estimation scheme introduced above, where the parameter $k$ adds a degree of freedom to the system and the observer guarantees the exponential convergence of the estimation error to a region around zero as well as the robustness with respect to the noises $\mu_{1}$ and $\mu_{2}$.

Proposition 2 Consider the UAV model described by (2)-(4). Assume that the variables $\eta_{2}, \nu_{2}, a_{\mu}$ are available for measurement and that Hypothesis 1 holds. Then for all $k \in \mathbb{R}^{+}, \alpha \in \mathbb{R}^{+}, \beta \in \mathbb{R}^{+}, \gamma \in \mathbb{R}^{+}$, the dynamic observer described by (31), (32), (34), (38), (39), and (40) with state $M_{\mu}(t) \in \mathbb{R}^{3} \times \mathbb{R}^{3}$, $\rho_{\mu}(t) \in \mathbb{R}^{3}, Q_{\mu}(t) \in \mathbb{R}^{3} \times \mathbb{R}^{3}, \delta_{\mu}(t) \in \mathbb{R}^{3}, \xi_{\mu}(t) \in \mathbb{R}^{3}$ and $\hat{\nu}_{1}(t) \in \mathbb{R}^{3}$ is such that

$$
\left\|\nu_{1}-\hat{\nu}_{1}\right\|^{2} \leq g_{1} \exp \left(-\lambda_{2} t\right)+g_{2} \mu_{M}^{2}
$$

for suitable positive reals $\lambda_{2}, g_{1}, g_{2}$, where $\mu_{M}$ is given in (27), for any initial conditions $M_{\mu}(0) \in \mathbb{R}^{3} \times \mathbb{R}^{3}$, $Q_{\mu}(0) \in \mathbb{R}^{3} \times \mathbb{R}^{3}, \rho_{\mu}(0) \in \mathbb{R}^{3}, \delta_{\mu}(0) \in \mathbb{R}^{3}, \hat{\nu}_{1}(0) \in \mathbb{R}^{3}$ with $Q_{\mu}(0)>0$. 
Proof. System (33) complies with the hypotheses of Lemma 1 with $\sigma_{\mu}$ in place of $x$ and $\left[\mu_{1}(t), \mu_{2}(t)\right]$ in place of $u(t)$, noting that hypothesis (i) is trivially verified and point (ii) is a consequence of the boundedness of the matrices $M_{\mu}(t)$ and $N_{2}(t)$. Thus, by $(27), \sigma_{\mu}$ is exponentially attracted in a ball whose radius depends on $\mu_{M}$. This property implies that system (41) also complies with Lemma 1 with $\chi_{\mu}$ in place of $x(t)$ and $\left[\sigma_{\mu}(t), \mu_{1}(t)\right]$ in place of $u(t)$. In fact, (41) complies with hypothesis (i) with $-\left[\beta+\frac{k}{4} Q_{\mu}(t) Q_{\mu}^{T}(t)\right]$ in place of $A(t)$ and (ii) follows noting that $M_{\mu}^{T}(t)$ and $Q_{\mu}(t)$ are bounded, so that the vector $\chi_{\mu}(t)$ is exponentially attracted in a ball with radius depending on $\mu_{M}$. Notice that from Claim 4 we have that if $Q_{\mu}(t)>0$, then matrix $Q_{\mu}(t)$ is bounded and positive definite for all $t \geq 0$. Hence system (42) verifies the hypotheses of Lemma 1 with $\tilde{\nu}_{1}(t)$ in place of $x(t)$ and $\left[\chi_{\mu}(t), \mu_{1}(t)\right]$ in place of $u(t)$, leading finally to inequality (43).

\section{Simulation Results}

\subsection{Illustration of the adaptive observer performances}

In this section we illustrate the observer designed above to estimate the linear velocity of an UAV based on the measurable angles, angular velocities and linear accelerations. These simulations also illustrate the effect of the tuning parameters $\alpha, \beta$ and $\gamma$ on the observer performances. In all simulations the observer estimations have initial conditions set to zero, while the desired states are time varying (and different from zero at $t=0)$. In all simulations both observers track the desired time varying states, with different performances and noise rejections. We have used the following parameters values :

\begin{tabular}{|l|l|}
\hline$m=2.500 \mathrm{~kg}$ & $k_{T}=10^{-5} \mathrm{~N} \cdot \mathrm{s}^{2}$ \\
$l_{b}=23 \mathrm{~cm}$ & $I_{R}=100 \cdot 10^{-7} \mathrm{~kg} \cdot \mathrm{m}^{2}$ \\
$I_{x x}=22493 \cdot 10^{-7} \mathrm{~kg} \cdot \mathrm{m}^{2}$ & $I_{y y}=222611 \cdot 10^{-7} \mathrm{~kg} \cdot \mathrm{m}^{2}$ \\
$I_{z z}=325130 \cdot 10^{-7} \mathrm{~kg} \cdot \mathrm{m}^{2}$ & $k_{M}=9 \cdot 10^{-5} \mathrm{~N} \cdot \mathrm{s}^{2} \cdot \mathrm{m}$ \\
$u_{G}=0.032 \mathrm{~m}$ & \\
\hline
\end{tabular}

Consider the first version of the observer, that does not take into account the measurement noise. In Fig. 2 the angular speeds, the angles, and the accelerations are plotted.

We choose in our simulation an angular speed vector $\nu_{2}(t)$ that spans periodically a 3-dimensional surface, in particular a non-vanishing periodic orbit. By virtue of Claim 1, this choice guarantees that $\mathrm{PE}$ Hypothesis 1 is satisfied. The time history of the three speeds to be observed is shown in Fig. 3a, and observer estimates in Fig. 3b, while estimation errors going exponentially to zero are shown in Fig. 3c. 


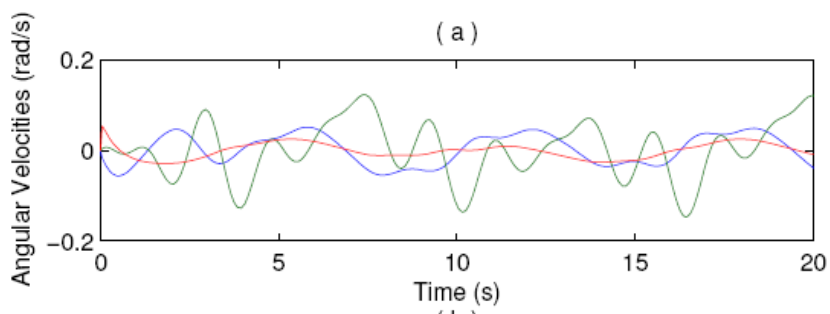

(b)

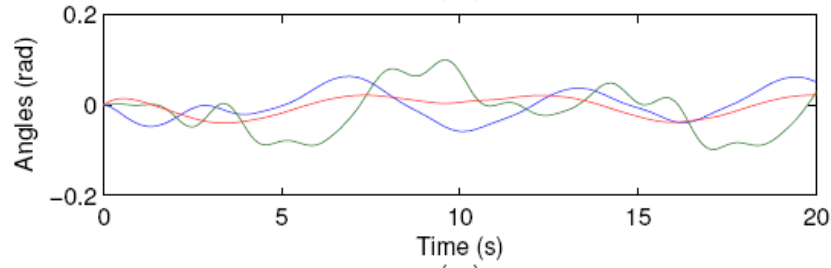

(c)

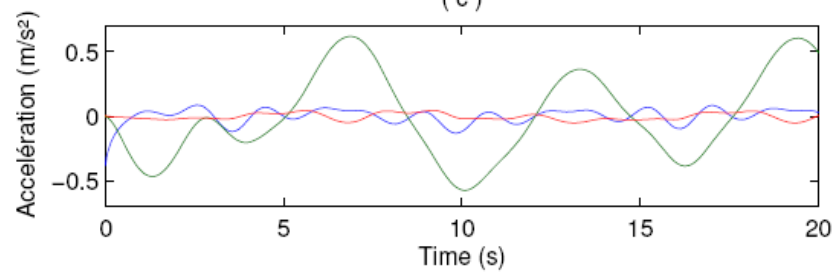

Figure 2: (a) Angular Speed, (b) Angles, (c) Acceleration

(a)

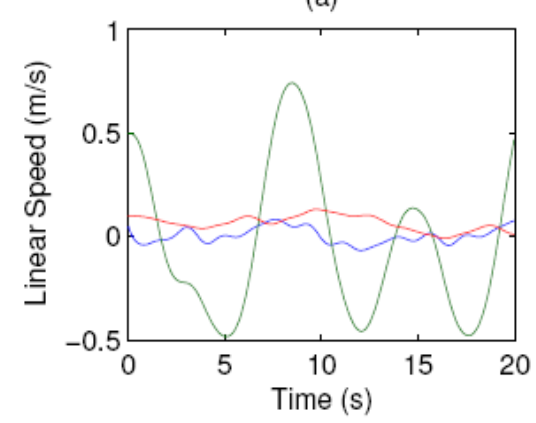

(c)

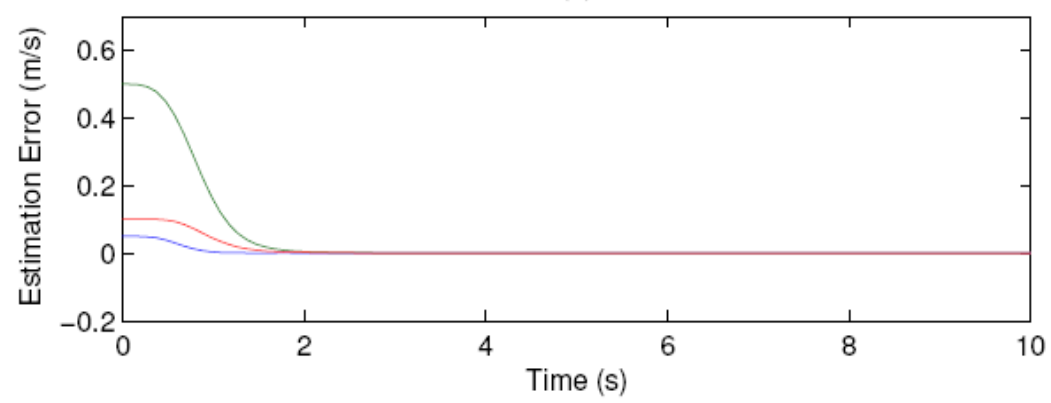

Figure 3: (a) Linear Speed, (b) Speed Estimation, (c) Estimation Error

We have then considered the first observer in the case of additive measurement noise. The measured acceleration is presented in Fig. 4 where the noise $\mu$ is 10 percent of the measured acceleration. 


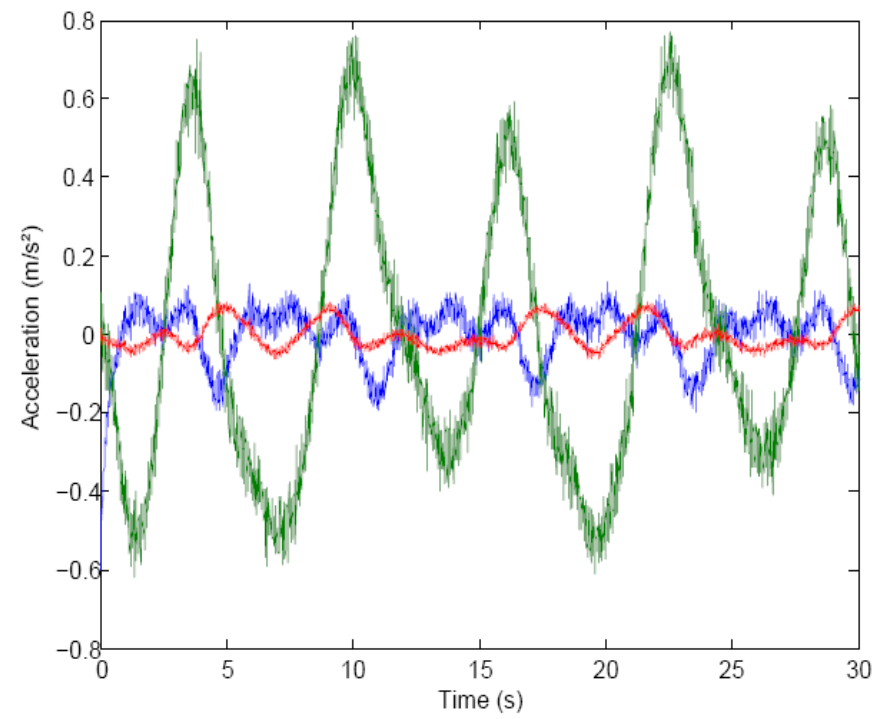

Figure 4: Acceleration

The simulation is undertaken using the second version of the observer, where the disturbance is attenuated following the design parameter $k$. A correct choice of parameter $\beta$ (which guarantees that $c_{2}=\lambda_{\min }\left(Q_{\mu}\right)$ is sufficiently big) also improve the convergence speed of the observer. One may see in Fig. 5a the velocity estimated by the observer, while Fig. 5b describes the estimation time history, and Fig. 5c illustrates the observation error going exponentially to a residual set given by the noise amplitude.

(a)

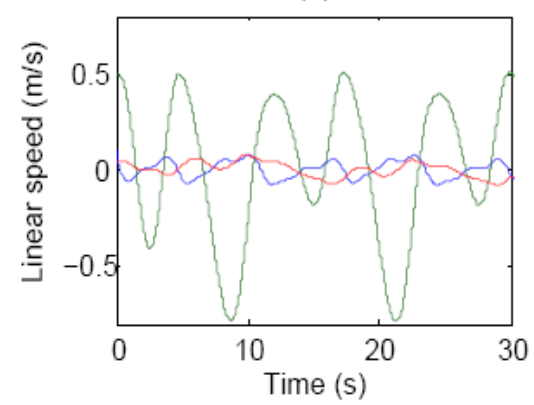

(c)

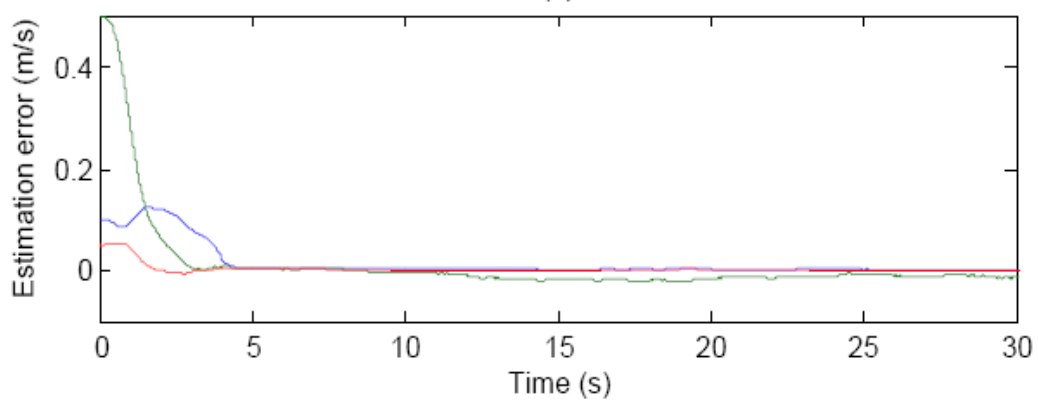

(b)

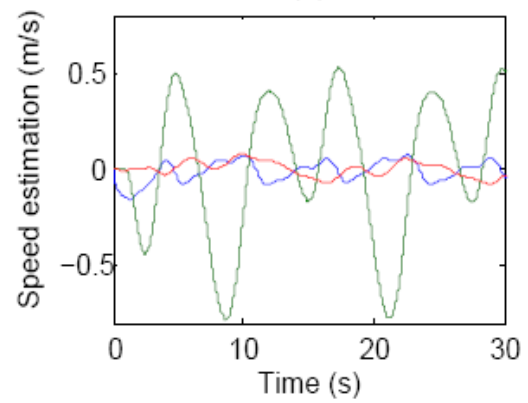

c) 


\subsection{Comparison with an Extended Kalman Filter}

The aim now is to compare the observer proposed in Section 3.2 with an Extended Kalman Filter (EKF), which is an established benchmark for estimation techniques. The EKF described here is based on the dynamic model of the UAV given by the continuous-time equations (2)-(4). We considered the corresponding discrete time model that can be written as

$$
\left\{\begin{array}{l}
X_{k+1}=f\left(X_{k}, U_{k}, W_{k}\right) \\
Y_{k}=h\left(X_{k}, Z_{k}\right)
\end{array}\right.
$$

The discrete-time state vector is $X_{k}=\left[\phi_{k}, \theta_{k}, \psi_{k}, p_{k}, q_{k}, r_{k}, u_{k}, v_{k}, w_{k}\right] . U_{k}$ is the control input. $W_{k}$ and $Z_{k}$ respectively represent process noise and measurement noise at time step $k$; they are zero mean white Gaussian sequences of random variables.

The behavior of the EKF designed to estimate the linear velocity of an UAV based on the measurable angles, angular velocities and linear accelerations is illustrated by numerical simulation and compared to the estimate provided by the observer described in Section 3.2. In all simulations, the observer estimations have initial conditions set to zero, while the desired states are time varying (and different from zero at $t=0)$. Model parameters are the same as those presented in Section 4.1 and we have considered additive measurement noise on the acceleration (see Fig. 5). First, we compare the performance of our estimation strategy with EKF by setting $k=50$, reporting the results in Figure 6 .
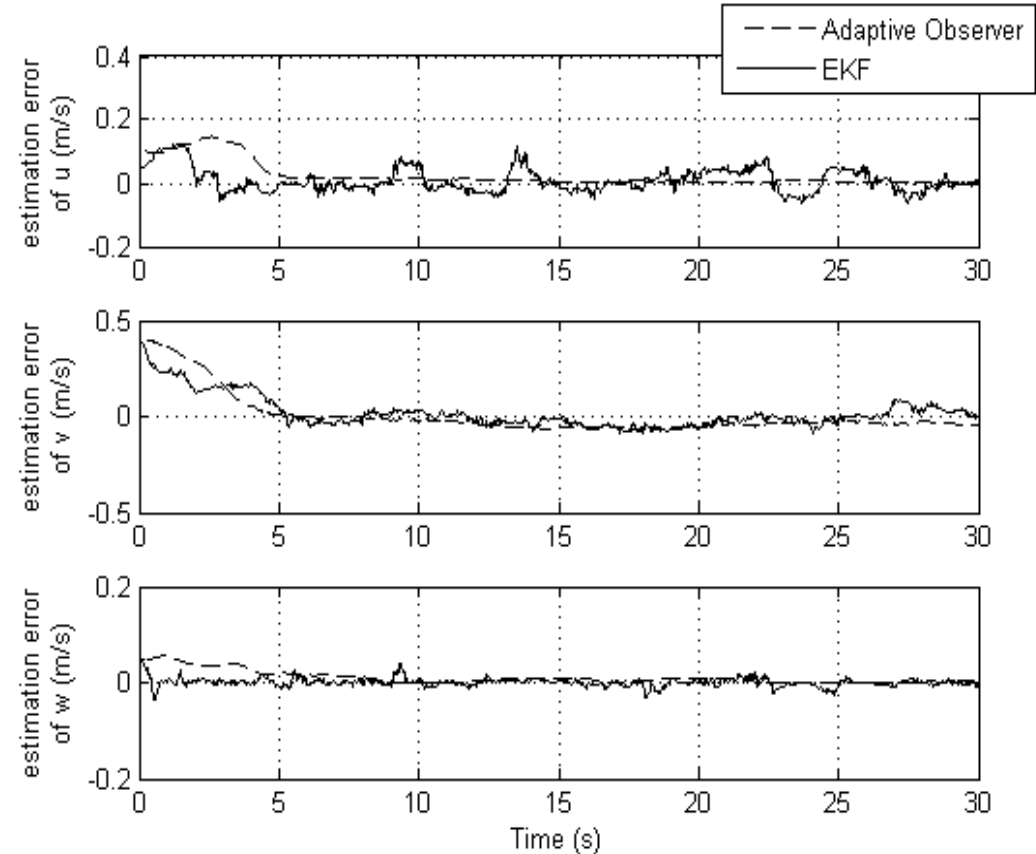

Figure 6: Comparison of the performances of the EKF and the Adaptive Observer for $k=50$.

They show that the algorithm in Section 3.2 significantly outperforms the EKF, since it guarantees in steady state condition a much smaller estimation error. Notice in this simulation (with $k=50$ ), the estimation error convergence of the algorithm in this note is slower than EKF; this is reasonable, since our strategy involves a cascade of filters, the transient performance of the entire algorithm being the sum of transient performances of the filters in the cascade. However, the total number of tuning parameters in our estimator is smaller than the number of parameters to be set in the EKF. As a consequence, the tunning procedure of our algorithms, verified in our simulations, is much more simple than that of the EKF. This is easily explained first by the small number of parameters, btheirut as weel as their clear effect on the convergence (through the analysis of the Lyapunov fonction). Secondly, the several dynamical variables behave as an automatic gain, this characteristic comming from adaptive theory make our observers rather self tunning compared to the EKF. 

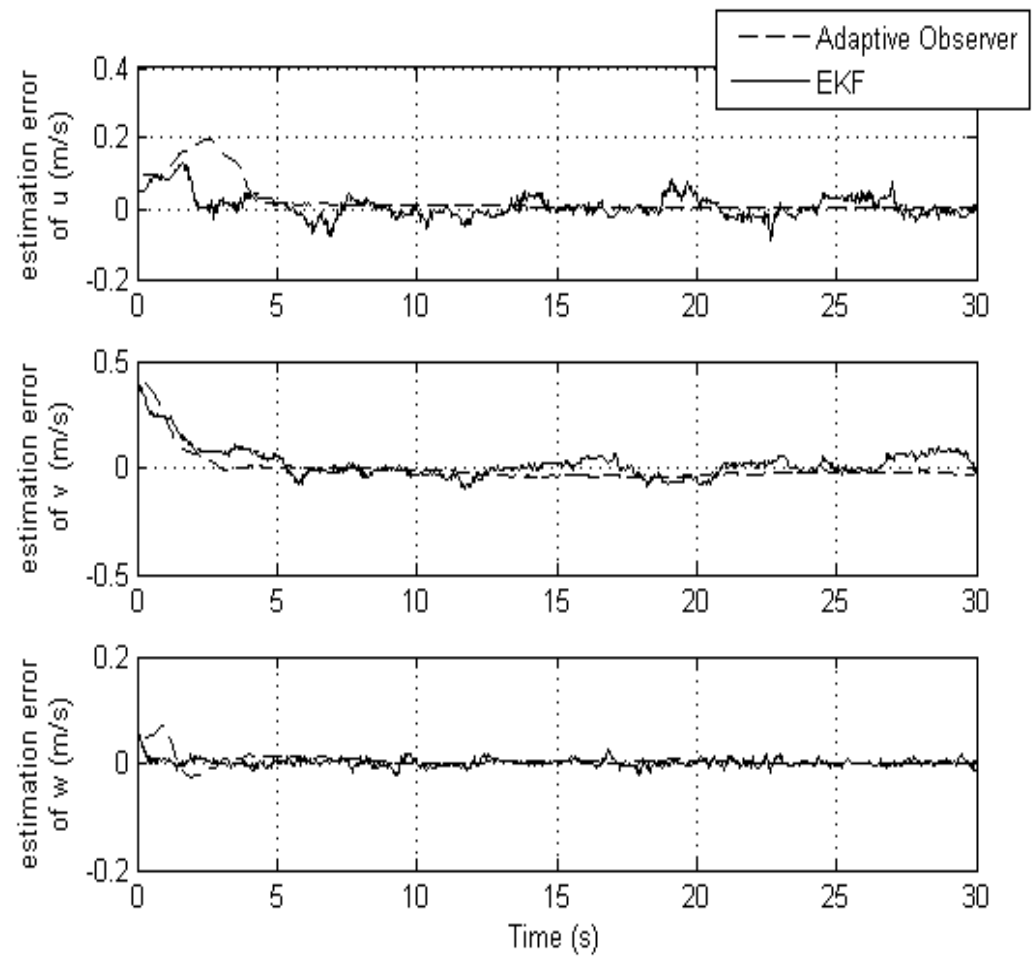

Figure 7: Comparison of the performances of the EKF and the Adaptive Observer for $k=10$.

By decreasing the value of $k$, in particular by setting $k=10$ and performing the simulation for this setting, we have a faster convergence rate, as displayed in Figure 7. Finally, Figure 8 shows that by choosing $k=0$, we obtain a steady state estimation error increase.
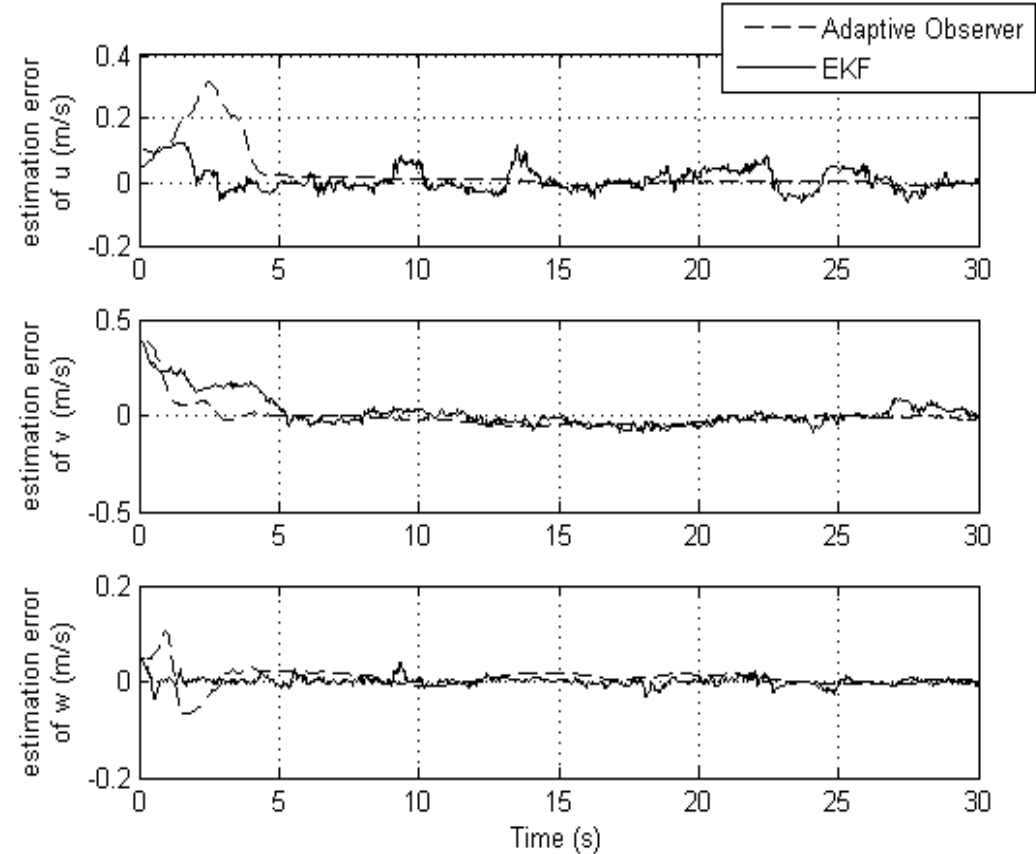

Figure 8: Comparison of the performances of the EKF and the Adaptive Observer for $k=0$.

This phenomenon can be explained as follows: > From expression (34) we infer that the bigger is $k$, 
the smaller is the norm of $Q_{\mu}$; the latter quantity in turn is related, by virtue of (42), to the inverse of the estimation error convergence rate. Thus by decreasing $k$ we may obtain a faster convergence rate but may have a greater sensibility to disturbances also; this property is inherently associated with a high gain estimation strategy.

In conclusion, the simulation tests show that the best performance on the algorithm in Section 3.2 are obtained via a fine tuning of the parameter $k$ in order to guarantee a small steady state estimation error outperforming EKF and simultaneously a convergence rate typical of a classic EKF approach.

\section{Conclusion}

In this work the UAV drone speed estimation problem has been addressed when no GPS related device is available. This is a realistic feature in many practical applications and has motivated recent works on the field. The results established for the specific prototype in this study hold also for the entire class of UAV such that rotational and translational dynamics may be decoupled, as helicopters and VTOL aircrafts.

In this context, the first theoretical issue arises by the fact that the linearized system is non-observable at the equilibrium point. Thus a nonlinear observability verification is performed for persistently exciting trajectories so that under some mild sufficient conditions an estimation strategy is shown to guarantee exponential estimation of the translational speed, by using the tools of adaptive observation theory.

A modified scheme is also proposed in order to obtain enhanced robustness property with respect to measurement noise. The theoretical results are illustrated via computer simulations, stressing the noise attenuation features. They also provide useful insight about the parameter tuning effect to achieve a satisfactory observer performance. Furthermore, computer simulations are used to compare the proposed estimation strategy with the classical Extended Kalman Filter. They show that the adaptive observer guarantees good performances even in presence of measurement noise, with greater disturbances attenuation with respect to the one provided by the Extended Kalman Filter.

Future research will be dedicated to the design of a simplified algorithm to possibly trade off noise immunity against faster convergence along with coupling the proposed observer to a control system designed to stabilize the 4-rotors helicopter. Additional work will be to implement the considered scheme in the UAV available at IBISC laboratory and to extend current results to the standard acceleration measurements provided by low cost IMUs.

\section{References}

[1] K. Benzemrane, "Développement d'observateurs et application au drone XSF", Ph.D. Thesis , Université d'Evry-Val d'Essonne, Evry, France, 2009.

[2] Azouz, N., Benzemrane, K., Damm, G., and Pradel, G., "Modeling and development of a 4rotors helicopter UAV", 6th IFAC Symposium on Intelligent Autonomous Vehicles, Toulouse, France, 3-5 Septembre, $200 \%$.

[3] A. Benallegue, A. Mokhtari and L. Fridman, "High-order sliding mode observer for a quadrotor UAV", Int. J. of Robust and Nonlinear Control, 2008.

[4] S. Bonnabel, P. Martin, and P. Rouchon, "A non-linear symetry-preserving observer for velocityaided inertial navigation", American Control Conference (ACC06), 2006, pp. 2910-2914

[5] G. Baldwin, R. Mahony, J. Trumph, T. Hamel, and T. Cheviron, "Complementary filter design on the special Euclidian group SE(3)"', European Control Conference (ECC 2007), 2007, pp. 37633770 .

[6] Benzemrane, K., G.L. Santosuosso, G. L., and Damm, G., "Unmanned aerial vehicle speed estimation via nonlinear adaptive observers", IEEE American Control Conference, July 2007.,pp. 985-990.

[7] L. Besnard, Y.B. Shtessel and B. Landrum, "Control of a quadrotor vehicle using sliding mode disturbance observer", American Control Conference, New York, 2007.

[8] M. Boutayeb, E. Richard, H. Rafaralahy, H.S. Ali and G. Zaloylo, "A simple time-varying observer for speed estimation of UAV", 17th IFAC World Congress, Seoul, Korea, 2008. 
[9] P. Castillo, A. Dzul, R. Lozano, "Real-time stabilization and tracking of a four-rotor mini rotorcraft", IEEE Transaction on control systems technology, vol. 12, pp. 510-516, July 2004.

[10] N. Guenardt, T. Hamel, V. Moreaut, "Dynamic modeling and intuitive control strategy for an "X4flyer" ", International Conference on Control and Automation (ICCA2005), June 27-29, 2005, Budapest, Hungary MM-4.4.

[11] T. Hamel and R. Mahony, "Image based visual servo control for a class of aerial robotic systems", Automatica, volume 43, number 11, pp. 1975-1983, $200 \%$.

[12] B. Herisse, F.X. Russotto, T. Hamel, R. Mahony., "Hovering flight and vertical landing control of a VTOL Unmanned Aerial Vehicle using Optical Flow",itInternational Conference on Intelligent Robots and Systems, 2008.

[13] A. Isidori, "Nonlinear Control Design - Geometric, Adaptive and Robust", Springer-Verlag Berlin, Heidelberg, Germany, 1989.

[14] H. Khalil, "Nonlinear Systems", $3^{\text {rd }}$ edition, Prentice Hall, New Jersey, 1996.

[15] R. Lozano, A. Dzul, P. Castillo, "Global stabilization of the PVTOL : Real-time application to a mini-aircraft", vol. 77, number 8, pp. 735-740, May 2004.

[16] F. LeBras, T. Hamel, and R. Mahony, "Nonlinear observer-based visual control of a VTOL UAV", European Control Conference, Kos, Greece, $200 \%$.

[17] J. Langelaan and S. Rock, "Passive GPS-free navigation for small UAVs", IEEE Aerospace Conference, pp. 1-9, Big Sky, Montana, March 5-12, 2005.

[18] T. Madani and A. Benallegue, "Sliding mode observer and backstepping control for a quadrotor unmanned aerial vehicles", American Control Conference, New York, $200 \%$.

[19] R. Marino, G. L. Santossuosso, P. Tomei, "Robust Adaptive Observers for Nonlinear Systems with Bounded Disturbances", IEEE Transactions on automatic control, Vol. 46, No 6, pp 967-972, June 2001.

[20] R. Marino, P. Tomei, Nonlinear Control Design - Geometric, Adaptive and Robust, Prentice Hall, Hemel Hempstead, London, 1995.

[21] N. Metni and T. Hamel, "A UAV for bridge inspection: Visual servoing control law with orientation limits", in journal of Automation in Construction, volume 17, number 1, pp. 3-10, $200 \%$.

[22] H. Nijmeijer and A. J. van der Shaft, Nonlinear Dynamical Control Systems New York: SpringerVerlag, 1990.

[23] J.M. Pflimlin, T. Hamel, P. Soueresand N. Metni, "Nonlinear attitude and gyroscope's bias estimation for a VTOL UAV", itInternational Journal of Systems Science, volume 38, number 3, pp. 197-210, $200 \%$.

[24] P. Pounds, R. Mahony, P. Hynes, J. Roberts, "Design of a Four-Rotor Aerial Robot", Procedings of 2002 Australasian Conference on Robotics and Automation, Auckland, 27-29 November 2002.

[25] G. Pradel, K. Benzemrane, G. Damm, N. Azouz "Modeling and development of a quadrotor UAV." Procedings of the 7th European Micro Air Vehicule Conference, Toulouse, September 17-21, 2007.

[26] D. Vissiere, P.J. Bristeau, A.P. Martin and N. Petit, "Experimental autonomous flight of a smallscaled helicopter using accurate dynamics model and low-cost sensors",17th IFAC World Congress, Seoul, Korea, 2008.

[27] D. Vissiere, A. Martin and N. Petit, "Using magnetic disturbances to improve IMU-based position estimation", European Control Conference, Greece, $200 \%$.

[28] D. Vissiere, A. Martin and N. Petit, "Using distributed magnetometers to increase IMU-based velocity estimation into perturbed area", 46th IEEE Conference on Decision and Control,200\%. 
[29] Vik, B. and Fossen, T.I., "A nonlinear observer for GPS and INS integration", Proceedings of the 40th IEEE Conference on Decision an Control, Orlando, Florida, USA, 2001

[30] Zhao, Y., and Slotine, J.J., "Discrete nonlinear observers for inertial navigation", Systems and Control Letters, 2005. 